\title{
MOTIVACIÓN HACIA LA QUÍMICA
}

\author{
DE MORÁN, J.A. ${ }^{1}$, DE BULLAUDE, M.E.G. ${ }^{2}$, y DE ZAMORA, M.M.K. ${ }^{1}$ \\ Facultad de Bioquímica, Química y Farmacia. \\ 2 Facultad de Agronomía y Zootecnia. \\ Universidad Nacional de Tucumán.
}

\section{SUMMARY}

The attitude towards a discipline is decisively important for learning, especially for difficult subjects, such as Chemistry.

In this paper we have intented to evaluate, into two groups of universitary students, the attitude towards Chemistry, the determinant factors, and the relationship between the attitude and the rank of knowledge of basic chemistry. Taking the results as a basis we propose methodologies to improve the development of favourabie attitude towards Chemistry.

\section{INTRODUCCIÓN}

Frecuentemente, los alumnos de asignaturas del área química de carreras universitarias emiten opiniones que evidencian su actitud negativa hacia el estudio de las mismas: «en realidad no necesitamos tanta química»; «los primeros años de la carrera son los peores; tenemos mucha química».

La actitud adversa observada es consecuencia de distintos factores entre los cuales pueden señalarse los siguientes:

- La brecha entre la madurez mental del alumno y el nivel de abstracción que demanda la disciplina conduce al estudiante a considerar que la química es aburrida, difícil y poco atractiva.

- Muchos profesores secundarios de ciencias físicoquímicas no están preparados para ejercer su difícil tarea, por carecer de formación en los aspectos pedagógicos y didácticos. Como consecuencia, crean en el alumno una imagen distorsionada de las asignaturas a su cargo.

La actitud hacia una disciplina reviste una decisiva importancia para el logro de la necesaria motivación en el aprendizaje de la misma, en especial cuando presenta las dificultades propias de la química. Por tal motivo, se impone un análisis de la incidencia relativa de un cierto grado de «aversión» hacia la química, y de sus posibles causas.

En el presente trabajo se ha intentado evaluar, en dos grupos de alumnos universitarios, las actitudes hacia el aprendizaje de la química, los factores que las determinan, y la relación entre la actitud y el nivel de dominio de química básica.

\section{MOTIVACIÓN}

Gagné (1975) sostiene que para que se produzca el aprendizaje es necesario un individuo motivado. La motivación, o sea la fuerza que impulsa al alumno hacia el aprendizaje, es uno de los problemas más complejos que debe enfrentar el docente de cualquier nivel. Puede definirse como una condición del organismo, cuya energía origina determinadas conductas, relacionadas con el ambiente para alcanzar un fin significativo. 
De parte del educador, motivación es el esfuerzo consciente realizado para establecer en los alumnos motivos que impulsen una actividad sostenida hacia las metas del aprendizaje. Motivo es una tendencia en actividad por encima del nivel de la acción refleja consciente.

Uno de los principales factores de la motivación es la actitud, que puede definirse como un estado motivacional perceptual que dirige la acción.

Según Gagné (1975), las actitudes son estados internos adquiridos, que ejercen influencias sobre la elección de la acción personal hacia cosas, personas o acontecimientos.

\section{EVALUACIÓN DE ACTITUDES}

Las actitudes poseen algunas propiedades que son importantes para el presente estudio:

a) Dirección: la actitud está dirigida hacia un objeto dado, una persona, una cosa, una ciencia, una profesión.

b) Magnitud: la intensidad de la actitud se distribuye a lo largo de un continuo que va desde el extremo más favorable al más desfavorable.

c) Centralidad: es la importancia que una actitud tiene con respecto a otras.

d) Multiplicidad: es la cantidad de factores que influyen en la actitud; la complejidad de la evaluación de la actitud es tanto mayor cuanto mayor sea el número de factores a considerar.

e) Modificabilidad: es el grado de posibilidad de modificar una determinada actitud.

La forma más corriente para la evaluación de actitưdes es el empleo de escalas preparadas al efecto (Rodríguez Kauth 1977).

En el presente trabajo se aplicó la técnica de Likert, que consiste en elaborar una lista de ítems o afirmaciones, entre las cuales se seleccionan veinte o treinta que se estima que se ajustan al propósito de la medición. Las afirmaciones deben contener ítems favorables y desfavorables hacia el objeto de la actitud, a fin de evitar la estereotipia de emitir en todos los casos la misma respuesta. Se brindan en el presente caso cuatro posibilidades. No se incluye una posibilidad intermedia para no proporcionar una forma de escape al compromiso de emitir una respuesta concreta.

La magnitud de la actitud se expresó en una escala con una valor mínimo de 0 , que corresponde a una actitud francamente negativa, y un valor máximo de 3 , que corresponde a una actitud francamente positiva. A los fines de la evaluación se adjudicó, para cada pregunta, puntajes $0-1-2-3$ a las distintas respuestas, asignando siempre el valor 0 a la respuesta más desfavorable y 3 a la más favorable.
Los ítems seleccionados para la encuesta se refieren a aspectos muy diversos, tales como:

- naturaleza de la química;

- preferencias de los alumnos;

- relación de la química con la carrera y con la vida diaria;

- características del curso y de los profesores.

Se intenta, mediante la selección de ítems sobre temas tan diversos, posibilitar la evaluación de la importancia relativa de los distintos factores que interactúan en el proceso de la formación de las actitudes. EI cuestionario correspondiente se detalla en el apéndice I.

\section{PRUEBA DE NIVEL}

A fin de determinar la relación existente entre la actitud hacia la química y el nivel de dominio de esta disciplina, se aplicó, junto con la encuesta de evaluación de actitudes, una prueba de nivel (detallada en el apéndice II) sobre aspectos básicos de química.

Debe hacerse notar que la formación previa de los ingresantes a las universidades argentinas es heterogénea, en especial en las disciplinas del área físico-química. Por tal motivo, se incluyen, necesariamente, entre los contenidos de química básica, nomenclatura (formulación) y estequiometría, y otros temas que evidentemente deberian ser conocidos por el alumno antes del ingreso a la universidad.

\section{APLICACIÓN DE LOS INSTRUMENTOS DE EVALUACIÓN}

La encuesta y la prueba de nivel se aplicaron a dos grupos de estudiantes que cursaban Química Analítica, asignatura del segundo año de sus respectivas carreras, y que en el cuatrimestre anterior habían completado los trabajos prácticos de Qúmica General e Inorgánica. Uno de los grupos (A) provenía de una facultad para cuyas carreras la química es sólo una de las materias básicas; el otro (B), de las Facultades de Bioquímica, Química y Farmacia, carreras que pertenecen al área química.

Los resultados correspondientes figuran en las tablas I y II.

\section{ANÁLISIS DE LOS RESULTADOS}

\section{Prueba de nivel}

- Las preguntas 1 (nomenclatura) y 6 (balanceo de una ecuación muy sencilla de óxido-reducción) tienen para 
Tabla I

Evaluación de actitudes.

Valores promedio.

\begin{tabular}{|ccc|}
\hline & GRUPO A & GRUPO B \\
1 & & \\
2 & 1,30 & 1,68 \\
3 & 1,01 & 1,22 \\
4 & 2,03 & 2,51 \\
5 & 2,18 & 2,72 \\
6 & 1,32 & 1,17 \\
7 & 1,58 & 1,81 \\
8 & 2,28 & 2,37 \\
9 & 0,29 & 1,82 \\
10 & 1,99 & 2,21 \\
11 & 1,98 & 2,52 \\
12 & 1,84 & 2,03 \\
13 & 2,00 & 2,12 \\
14 & 0,96 & 0,92 \\
15 & 1,93 & 2,00 \\
16 & 1,93 & 2,47 \\
17 & 1,53 & 1,53 \\
18 & 1,52 & 1,59 \\
19 & 1,59 & 1,50 \\
20 & 1,39 & 2,29 \\
\hline
\end{tabular}

$B$ un alto porcentaje de respuestas, lo que es de esperar dado el nivel de estos ítems; y para $\mathrm{A}$ un $50 \%$ del correspondiente a $\mathrm{B}$.

- El ítem 9 (equilibtio químico) es evidentemente bastante sencillo para A y $\mathrm{B}$, dados los porcentajes obtenidos.

- Para la pregunta 2 (gases ideales), la mayoría de los alumnos de $\mathrm{A}$ y de $\mathrm{B}(60 \%)$ escriben la ecuación de Boyle; sólo el $20 \%$, la ecuación de estado, pese a que el tema ha sido tratado también en física.

- Para el ítem 8 (soluciones) se podría esperar mejores resultados.

- El ítem 7, referente a la teoría de Lowry-Bronsted, es respondido por menos de la tercera parte de los alumnos.

- La mayoría de los estudiantes no intenta responder a los sencillos problemas de estequiometría formulados en las preguntas 3 y 10 , lo que evidencia las dificultades que este tema plantea al alumno.

- Los resultados obtenidos para el f́tem 4 demuestran para ambos grupos poco conocimiento de la tabla periódica.
Tabla Il

Prueba de nivel.

Porcentaje de respuestas correctas.

\begin{tabular}{|rcc|}
\hline & GRUPO A & GRUPO B \\
1 & & \\
2 & 58 & 92 \\
3 & 59 & 62 \\
4 & 10 & 8 \\
5 & 6 & 14 \\
6 & 1 & 8 \\
7 & 41 & 84 \\
8 & 23 & 34 \\
9 & 26 & 58 \\
10 & 68 & 81 \\
\hline
\end{tabular}

- La pregunta 5, sobre estructura de Lewis, es la que presenta menor porcentaje de respuestas correctas.

Las calificaciones promedio obtenidas en la evaluación son en general, superiores para el grupo B que para A. EI promedio general es de $45,6 \%$ para $\mathrm{B}$ y $30,7 \%$ para $\mathrm{A}$. Por otra parte, sólo el $46 \%$ de los estudiantes del grupo $B$ y el $10 \%$ de los del grupo A obtienen en la prueba calificaciones superiores a $5(50 \%)$. El resultado para B es muy bajo para carreras en las cuales el eje principal es la química.

\section{Actitudes}

El promedio general obtenido en la evaluación de actitudes fue 1,64 para el grupo A y de 1,93 para el grupo B.

La temática a la que hace referencia cada pregunta se consideró arbitrariamente como de influencia positiva o negativa cuando los valores obtenidos para el grupo en cuestión son, respectivamente, superiores o inferiores al promedio general obtenido para el mismo.

Algunos factores que sin duda alguna ejercen influencia positiva, de acuerdo con los resultados obtenidos, son Ios siguientes:

- relevancia de la química para las respectivas carreras (ftems 3 y 4 );

- relación de la química con la vida diaria (ítem 12);

- las prácticas de laboratorio (ítem 10).

Factores que evidencian una neta influencia negativa son:

- Ias dificultades propias de la disciplina (ítems 2 y l3); 
- la exigencia por parte de los profesores (ítem 5);

- aversión a algunos temas: nomenclatura (ítem 1) y problemas (item 18).

Son contradictorias las respuestas referentes a las clases de la materia: en el f́tem 7 parecen demostrar que el alumno entiende las clases, péro según los resultados del ítem 17, las mismas no son claras; el análisis conjunto de los ítems 6 y 11 tampoco permite llegar a una conclusión. Según las respuestas de los íterns 14 y 20 , los estudiantes están atentos durante las clases, aunque, de acuerdo con el ftem 19 hay una diferencia fundamental para las distintas facultades en cuanto a la actitud hacia las mismas.

La influencia de algunos factores difiere según la carrera. Por ejemplo, para el ítem 8 , que pregunta si la elección de la carrera estuvo basada en el gusto por la química, el resultado para el grupo A, como era de esperar, es el valor más bajo obtenido para este grupo. Resulta sorprendente, en cambio, que para B este ítem obtenga resultados inferiores al promedio.

Es destacable la falta de relación entre las respuestas referentes a las clases y a la disposición para el estudio. El alumno promedio, pese a afirmar que atiende en las clases, que comprende lo que se explica en ellas, manifiesta que la química no es fácil y que estudiar química le cuesta.

\section{CORRELACIÓN ENTRE ACTITUD Y NIVEL DE DOMINIO}

La correlación obtenida entre el nivel de actitudes y de dominio de la asignatura es 0,22 .

\section{Discusión de los resultados}

Es importante destacar la baja corrclación entre el nivel de actitudes y de conocimientos. Aparentemente, un estudiante con actitud muy positiva hacia la química, que estaría fuertemente impulsado hacia su estudio, deberia haber alcanzado un buen nivel de conocimientos.

Sin embargo, para que una actitud sea consistente, sus tres componentes, cognitivo, afectivo y volifivo, deben tener la misma dirección (Rodriguez Kauth 1987).

El componente cognitivo, que se refiere a tos conocimientos que se tengan acerca del objeto de la actitud, está afectado por las percepciones de los alumnos respecto a las ciencias químicas. Es fundamental, en este aspecto, la influencia del docente. El profesor, en especial el que inicia al adolescente en el estudio de las ciencias, puede presentar la química y otras ciencias como disciplinas interesantes, pero incomprensibles para la mayoria, exigiendo memorización mecánica de temas como nomenclatura, leyes, etc.; o inducir al estudiante a razonar, demostrándole la accesibilidada esta ciencia.
Wall y Varma (1975) afirman que «la energía, el impulso del alumno, sus aspiraciones, su reacción ante las dificultades, pueden ser afectadas profundamente -para bien o para mal-por las creencias y la filosofía del maestro».

El componente volitivo conduciría a la acción, de acuerdo con las pautas fijadas por los otros componentes. Sin embargo, no siempre la acción responde a tales pautas, ya que en algunos casos existen demandas ocultas, provenientes de otra actitud más central e imperiosa. Tal es el caso del estudiante atraído hacia la química en el aspecto afectivo, pero que rechaza inconscientemente su estudio por temor al esfuerzo. Como afirma Sacristán Gómez (1982): «Por mucho que sea el interés suscitado por un estímuio, es preciso que se quiera, y que se quiera efectivamente, para ejecutar la acción que intelectualmente aparece como deseable, a la vista de las instancias didácticas del docente».

Debe destacarse que el sistema actual de enseñanza universitaria en la Argentina permite al alumno postergar durante varios años el momento de «enfrentarse a los libros» para rendir el examen final de la asignatura. Como consecuencia, se favorecen hábitos negativos: pereza, inconstancia y desorden intelectual.

En cuanto al bajo nivel de conocimientos alcanzado por el grupo en estudio, podría ser explicado por la influencia de los siguientes factores:

- Las dificultades propias de la química, disciplina que combina la abstracción con la abundancia de material fáctico.

- La falta de una adecuada metodología de estudio. Según Johnstone (1984), frecuentemente «los métodos por los cuales los estudiantes aprenden están en conflicto o con la naturaleza de la ciencia o con los métodos tradicionales de enseñanza».

- El escaso interés de la mayoría de los profesores por formar a sus alumnos, ayudándoles a adquirir buenos hábitos de trabajo intelectual.

\section{Propuestas}

- Es indispensable enfatizar los aspectos de la disciplina que favorecen una actitud positiva hacia la misma, ya que, como afirma Carter (1989): «Las percepciones de los estudiantes sobre el contexto de los cursos de química influencian su aprendizaje». En especial para aquellas carreras en las cuales la química es sólo una de las materias básicas, es conveniente destacar aspectos motivantes para alumno y profesor, tales como: la relación de la química con la vida diaria, los temas referentes a la protección del medio ambiente o los que tengan conexión con los intereses vocacionales del estudiante, y las prácticas de laboratorio.

- El docente debe recordar que el conocimiento no puede ser transferido intacto desde su mente a la del alumno, ya 
que éste debe «construir» su propia estructura cognoscitiva, en función de esquemas preexistentes. Por lo tanto, una de las tareas fundamentales del profesor es enseñar a aprender, fomentando en el estudiante la adquisición de hábitos de estudio, ayudándole a utilizar el material bibliográfico, planteando, como parte de su curso, problemas reales cuya resolución exija al alumno obtener información y procesarla correctamente.

\section{REFERENCIAS BIBXIOGRÁFICAS}

CARTER, C.S.y BRICKHOUSE, N.W, 1989. Journal Chem. Education, 225, pp. 663 .

GAGNÉ, R., 1975. Principios básicos del aprendizaje para la instrucción. (Editorial Diana: Méjico).

IOHNSTONE, A.H., 1984. Journal Chem. Education, 61(10), pp. 847.
RODRIGUEZ KAUTH, A., 1987. Psicología de las actitudes yestructuras cognitivas, pp. 22-28. (Editorial Universitaria: San Luis, Argentina).

SACRISTÁN GÓMEZ, D., 1982. Revista Españolade Pedugogía, año $\mathrm{XL}$, núm. 156, pp. 85.

WALL, W.D.y VARMA, V.P., 1975. Avances en psicología de la educación. (Morata: Maórid).

\section{APÉNDICE I}

Evaluación de actitudes

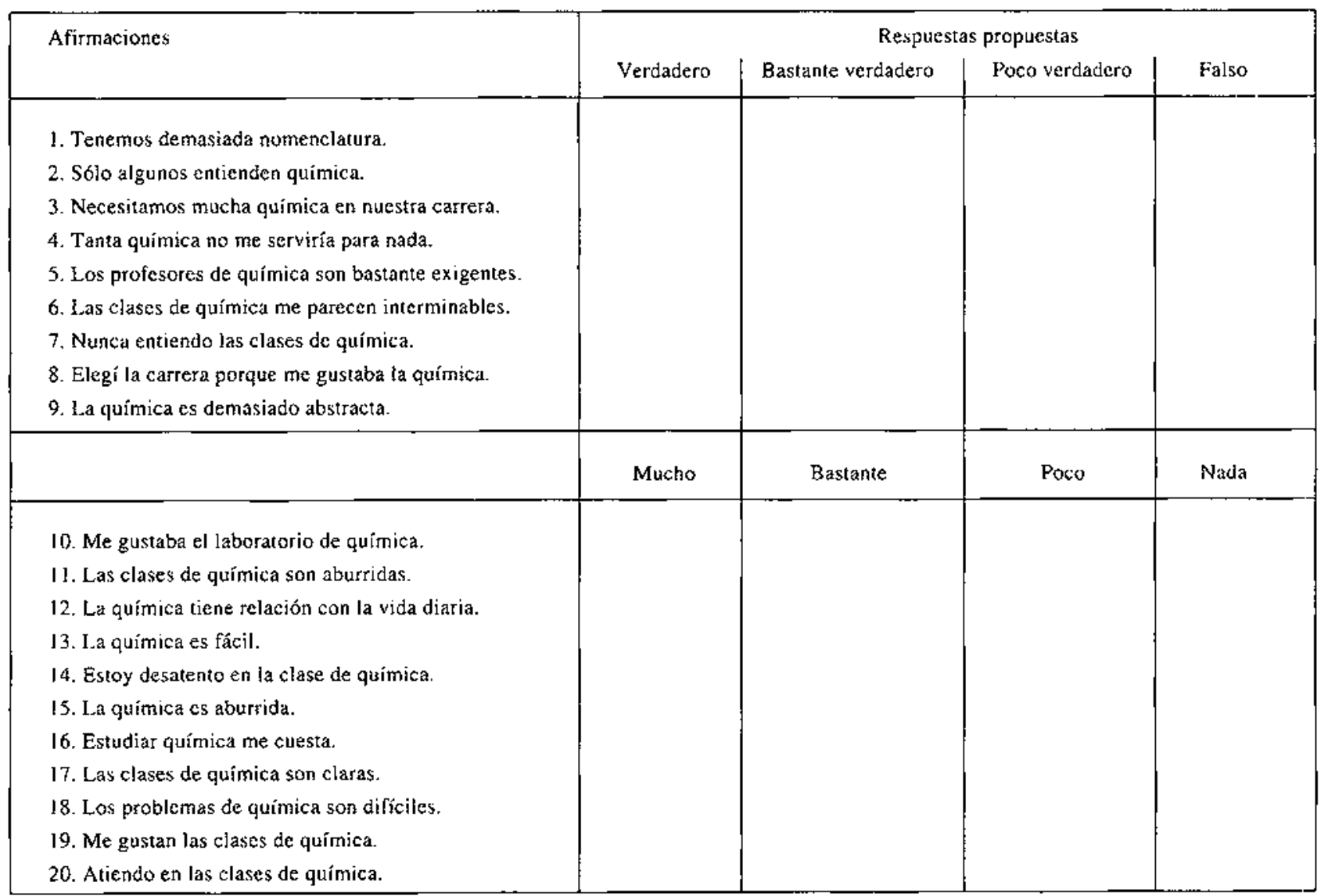




\section{APÉNDICE II}

Evaluación de nivel

Rindió Quimica Inorgánica? Edad Carresa

1) Escriba el nombre de:

$\mathrm{Mg}\left(\mathrm{H}_{2} \mathrm{PO}_{1}\right)_{2}$

$\mathrm{Na}_{2} \mathrm{SiO}_{3}$

$\mathrm{Al}_{2}\left(\mathrm{SO}_{1}\right)_{3}$

$\mathrm{Fe}(\mathrm{CIO})$ )

$\mathrm{KMnO}$

2) Eiscriba a) la cxpresión matemática de la Ley de Boyle

b) la ecuación de estado de los gases ideales.

3) Al arder Ig de vela en $\mathrm{O}_{2}$, el $\mathrm{CO}_{2}$ y vapor de agua resultante de la combustión pesan más de un gramo. Explique por qué.

4) Clasifique los siguienles elementos como representativos, de transición o de transición interna:

$\mathrm{Na}-\mathrm{N}-\mathrm{Zr}-\mathrm{As}-\mathrm{Xe}-\mathrm{Cu}-\mathrm{Ge}-\mathrm{He}-\mathrm{Se}$

5) Escriba la estructura de Lewis del $\mathrm{Ca}\left(\mathrm{No}_{3}\right)_{2}$

6) Iguale por óxido-reducción:

$\mathrm{HI}+\mathrm{H}_{2} \mathrm{SO}_{4} \Rightarrow \mathrm{SO}_{2}+\mathrm{I}_{2}+\mathrm{H}_{2} \mathrm{O}$

7) En la siguiente reacción indique cuáles son los ácidos y la bases conjugados:

$\mathrm{H}_{2} \mathrm{O}+\mathrm{CO}_{3}{ }^{2 \cdot} \Leftrightarrow \mathrm{HCOs}+\mathrm{OH}^{*}$

8) ¿Qué significa $\mathrm{H}_{2}, \mathrm{SO}_{4}$ al $98 \%$ ?

9) ¿Hacia dónde se desplaza el equilibrio, en la siguiente reacción, por agregado de CO?

$\mathrm{CO}_{(|;|)}+\mathrm{Cl}_{2[\mathrm{~g} \mid} \Leftrightarrow \mathrm{COCl}_{2\{\mathrm{k}]}$

10) En la reacción:

$5 \mathrm{~A}(\mathrm{~g})+3 \mathrm{~B}(\mathrm{~g}) \Rightarrow 2 \mathrm{C}(\mathrm{g})+4 \mathrm{D}(\mathrm{g})$

¿Cuántos $\mathrm{dm}^{2}$ de $C$ se forman a partir de $10 \mathrm{~cm}^{3}$ de $A$ ? 